\title{
Comparative Evaluation of Post-TURP Complications in Patients with BPH Presenting with and Without Acute Urinary Retention
}

\author{
Veda Murthy Reddy Pogula ${ }^{1}$ Ershad Hussain Galeti ${ }^{1}$ Ifrah Ahmad ${ }^{1} \quad$ Bhargava Reddy Kanchi ${ }^{1}$ \\ ${ }^{1}$ Department of Urology, Narayana Medical College, Nellore, Andhra \\ Pradesh, India \\ Address for correspondence Ershad Hussain Galeti, Department of \\ Urology, Narayana Medical College, Nellore 524003, Andhra Pradesh, \\ India (e-mail: dr.ershadhussain@gmail.com). \\ Int J Recent Surg Med Sci 2022;8:39-43.
}

\begin{abstract}
Keywords

- acute urinary retention

- benign prostatic hyperplasia

- lower urinary tract symptoms

- postvoid residual

Background Benign prostatic hyperplasia (BPH) is a prevalent urological condition affecting men at an older age. Acute urinary retention (AUR) is a severe symptom of men who develop BPH. TURP is the gold standard as the management of BPH is concerned. Our study tried to compare the post-TURP complications between patients presented with and without AUR.

Materials and Methods We enrolled 126 patients, out of which 74 were in the AUR group and 52 in the non-AUR group. The mean age of patients with AUR was 62.51 years, and that for patients without AUR was 61.06 years. Statistical significance was noted in our study in patients with AUR and without AUR regarding the prostate's grading by DRE, the volume of gland, PSA level, post-TURP UTI, recatheterization post TURP, length of hospital stays with $p$-values $0.000,0.000,0.006,0.004,0.007$, and 0.000 , respectively. Statistical significance was not noted in patients with AUR and without AUR with regard to the grading of hypertension, diabetes mellitus, ischemic heart disease, post-op TURP syndrome, post-TURP hematuria, patients needing a blood transfusion, post-TURP sepsis, LUTS, post-TURP stricture, resurgery for clot retention with $p$ values of $0.918,1.000,1.000,1.000,0.523,0.642,1.000,0.319,1.000$, and 1.000 , respectively.

Conclusion Our study shows that post-TURP complications such as hematuria, blood transfusion rate, post-op UTI, sepsis, recatheterization, lower urinary tract stricture, resurgery, TUR syndrome, and the length of hospital stay were higher in patients who presented with AUR than in those without AUR. Post-TURP UTI complications, recatheterization rate, and the length of hospital stay were statistically significant in the AUR group compared with the non-AUR group. Therefore, it is better to intervene earlier before the patients develop AUR to minimize the complications and maximize the outcomes.
\end{abstract}

DOI https://doi.org/ 10.1055/s-0041-1736446. ISSN 2455-7420.

\footnotetext{
(c) 2021. Medical and Surgical Update Society. All rights reserved. This is an open access article published by Thieme under the terms of the Creative Commons Attribution-NonDerivative-NonCommercial-License, permitting copying and reproduction so long as the original work is given appropriate credit. Contents may not be used for commercial purposes, or adapted, remixed, transformed or built upon. (https://creativecommons.org/ licenses/by-nc-nd/4.0/) Thieme Medical and Scientific Publishers Pvt. Ltd., A-12, 2nd Floor, Sector 2, Noida-201301 UP, India
} 


\section{Introduction}

Benign prostatic hyperplasia (BPH) is a prevalent urological condition affecting men at an older age. BPH remains one of the most common causes in men that can give rise to lower urinary tract symptoms, with or without bladder outlet obstruction (BOO). The pathological process in BPH is hyperplasia, which affects both the stromal and glandular elements of this gland. This condition affects the quality of life (QOL) significantly in many patients. Even though most seek medical intervention because of bothersome symptoms, BOO was found in $60 \%$ of symptomatic and $52 \%$ of asymptomatic patients. ${ }^{1,2}$ Intervention may be needed for bothersome symptoms in around $30 \%$ of those older than 65 years. $^{3}$ For males older than 50 years, TURP is the second most common surgery performed next only to cataract surgery. ${ }^{4}$ Even though many new management modalities for the BPH have been developed, transurethral resection of the prostate is still the gold standard as far as BPH management is concerned. ${ }^{5}$ LASER development in endourology is gradually replacing TURP in BPH management. Holmium laser (HoLEP) is the gold standard ${ }^{5,6}$ though many urologists have a reservation in accepting this as the gold standard. The major disadvantage is the prohibitive cost of these lasers. TURP has become a relatively safer procedure due to newer technologies in diathermy and visual scopes. But still, there is a chance of TURP syndrome and electrolyte imbalance, especially in high-risk cardiac patients. The risk is accentuated by the use of glycine for irrigation. The complication rates were decreased with the development of bipolar diathermy with normal saline as the irrigant fluid.

Acute urinary retention (AUR) is defined as a sudden and painful inability to void voluntarily. ${ }^{7,8}$ Even though there are many causes of AUR, the most common cause is BPH. The prevalence rate of AUR in men with BPH is estimated to be as high as $53 \%{ }^{9}$ AUR was the chief complaint in 20 to $42 \%$ of men who underwent TURP. ${ }^{10}$ Escalating postoperative complications and more extended hospital stay in men with $\mathrm{BPH}$ who develop AUR have been reported. ${ }^{10-12}$ Patients presented with AUR had a high mortality rate in the first 3 years after prostatectomy. ${ }^{13}$ Our study compared the post-TURP complications between patients presented with and without AUR.

\section{Materials and Methods}

We compared the TURP (monopolar) outcomes and complications for BPH patients with and without AUR.

Patients admitted to our institute with lower urinary tract symptoms (LUTS) due to BPH with and without AUR were included in the study. This was a prospective observational study conducted from January 2019 to December 2020. The Ethics Committee of this institution approved to conduct of this study. All men who participated in this study provided written consent.

The patients with complaints suggestive of LUTS were thoroughly evaluated with history and physical examination, digital rectal examination (DRE), ultrasound KUB (Kidney, ureter, urinary Bladder), post-void residual (PVR) urine, and patients with BPH were selected. Patients who presented with and without AUR were assigned as Group A and Group $B$, respectively. Inclusion criteria were prostate size $>30 \mathrm{gm}$ and less than $60 \mathrm{~g}$, maximum flow rate (Qmax) less than 10 $\mathrm{mL} / \mathrm{s}$, men more than 45 years old and less than 70 years old, PVR urine exceeding $100 \mathrm{~mL}$. The exclusion criteria were urethral stricture, neurogenic bladder, previous prostate or urethral surgery, prostate cancer, and unwilling patients.

Post-surgery Foleys catheter was removed on postoperative day 3 in all patients. Patients were discharged on their symptom-free comfort days as they had come from a very long distance and were from a rural background. Patients were reviewed 1-week post discharge for urine culture to look for postoperative urinary tract infection (UTI).

DRE grading was done by a single surgeon to avoid observer error. Classification-Grade I BPH on DRE was appreciated as easy accessibility of the upper limit of the prostate, about one finger width depth of the lateral sulcus; Grade II BPH as the accessibility of the upper limit of the prostate with little effort, $>$ one but $<$ two finger width depth of lateral sulcus, prominent median sulcus; Grade III as the accessibility of the upper limit of the prostate with marked difficulty, about two finger width depth of lateral sulcus, obliteration of median sulcus with a round posterior surface; and Grade IV as the inability to access the upper limit even with effort, deep depth lateral sulcus ( $>$ two finger width), obliteration of median sulcus with a round posterior surface. ${ }^{14}$

\section{Statistical Analysis}

Mean values, standard deviations, and percentage for the groups were computed. Results on continuous measurements are presented as mean and standard deviation (SD). Student's $t$-test was used to determine the statistical significance between the two group means. All analyses were twotailed, and $p<0.05$ was considered significant.

\section{Results}

We enrolled 126 patients, out of whom 74 were in the AUR group and 52 in the non-AUR group. Out of 126 patients, $34.9 \%$ were less than 60 years old, $34.1 \%$ were between 61 and 65 years of age, and $31 \%$ of patients were more than 65 years. In the non-AUR group, $36.50 \%$ of the patients were less than 60 years of age, 36.50\% were between 60 and 65 years, and $26.90 \%$ were more than 65 years old. In the AUR group, $33.80 \%$ of the patients were below 65 years of age, $32.40 \%$ were between 60 and 65 years, and $33.80 \%$ were more than 65 years. The mean age of patients with AUR was 62.51 years and that of patients without AUR was 61.06 years. The $p$-value of 0.711 was insignificant ( - Table $\mathbf{1}$ ).

In the current study, $21.6 \%$ of the patients with AUR had hypertension, and $19.2 \%$ of patients without AUR had hypertension. A $p$-value of 0.918 was insignificant ( - Table $\mathbf{1}$ ).

In the current study, $23.0 \%$ of the patients who presented with AUR had diabetes as a co-morbid condition, and $23.1 \%$ of the patients without AUR had diabetes (both groups had 
Table 1 Master table comparing all variables with $p$-values

\begin{tabular}{|l|l|l|l|l|}
\hline Sr. no & Variables & $\begin{array}{l}\text { With AUR } \\
(\%)\end{array}$ & $\begin{array}{l}\text { Without AUR } \\
(\%)\end{array}$ & $p$-Value \\
\hline 1 & HT & 21.6 & 19.2 & 0.918 \\
\hline 2 & DM & 23.0 & 23.1 & 1.000 \\
\hline 3 & IHD & 10.8 & 9.6 & 1.000 \\
\hline 4 & Volume (mean) & $53.20 \mathrm{~mL}$ & $44.21 \mathrm{~mL}$ & 0.000 \\
\hline 5 & PSA (mean) & 3.357 & 3.094 & 0.006 \\
\hline 6 & TUR syndrome & 1.4 & 0.0 & 1.00 \\
\hline 7 & Hematuria & 10.8 & 5.8 & 0.523 \\
\hline 8 & Blood transfusion & 4.1 & 1.9 & 0.642 \\
\hline 9 & UTI-post operative & 24.3 & 3.8 & 0.004 \\
\hline 10 & Sepsis & 1.4 & 0.0 & 1.000 \\
\hline 11 & Recatheterization & 23.0 & 3.8 & 0.007 \\
\hline 12 & Irritative LUTS & 24.3 & 15.4 & 0.319 \\
\hline 13 & Stricture & 2.7 & 1.9 & 1.000 \\
\hline 14 & Resurgery & 1.4 & 0.0 & 1.000 \\
\hline 15 & Length of stay (mean) & 7 & 4.56 & 0.000 \\
\hline 16 & PVR (mean) & 14.31 & 13.32 & 0.062 \\
\hline 17 & Qmax (mean) & 19.22 & 19.20 & 0.947 \\
\hline
\end{tabular}

Abbreviations: AUR, acute urinary retention, DM, diabetes mellitus; HT, hypertension; IHD, ischemic heart disease; LUTS, lower urinary tract symptoms; PVR, postvoid residual; PSA, prostate-specific antigen; Qmax, maximum flow rate; TUR, transurethral resection syndrome; UTI, urinary tract infection.

almost the same percentage $)(p$-value $=1.000)$. There was no statistical significance ( - Table $\mathbf{1})$.

In the current study, $10.8 \%$ of patients with AUR suffered from ischemic heart disease, whereas $9.6 \%$ of patients without AUR had ischemic heart disease. If the patient was on an anti-platelet drug, we asked them to stop it 1 week before the procedure. There was no statistical significance $(p=1.000)$ ( - Table $\mathbf{1}$ ).

Regarding the prostate grading by DRE, $9.5 \%$ of the patients with AUR and $40.4 \%$ without AUR had grade I enlargement. In addition, $89.2 \%$ of patients with AUR and $59.6 \%$ without AUR had grade II enlargement, $1.4 \%$ of patients with AUR and no patients without AUR had grade III enlargement. In our study, the $p$-value was 0.000 ; most patients with AUR had grade II enlargement of the prostate. In patients without AUR, they had grade I and grade II enlargement, with grade II outnumbered I ( - Table $\mathbf{2}$ ).

The gland volume serum PSA level was statistically significant between the two groups ( $p=0.000$ and 0.006 , respectively) (-Table $\mathbf{1}$ ).

Table 2 DRE grading

\begin{tabular}{|l|l|l|l|l|}
\hline & Grade I & Grade II & Grade III & Total \\
\hline With AUR $(n)$ & 7 & 66 & 1 & 74 \\
\hline Without AUR $(n)$ & 21 & 31 & 0 & 52 \\
\hline Total $(n)$ & 28 & 97 & 1 & 126 \\
\hline
\end{tabular}

Abbreviations: AUR, acute urinary retention; DRE; digital rectal examination.
TUR syndrome occurred only in one patient in the AUR group at the end of surgery. Serum electrolytes were evaluated, which showed dilutional hyponatremia $(p=1.000)$ (-Table 1).

In the current study, $10.8 \%$ of patients presenting with AUR had hematuria after TURP, whereas $5.8 \%$ of patients without AUR had hematuria ( $p=0.523$, not statistically significant) (-Table $\mathbf{1}$ ).

In the present study, $4.1 \%$ of patients in the AUR group needed a blood transfusion due to persistent hematuria, whereas $1.9 \%$ of patients without AUR needed a blood transfusion ( $p=0.642$, not statistically significant) ( - Table $\mathbf{1}$ ).

Sepsis was seen in only one patient in the AUR group, who was appropriately treated with higher antibiotics. There was no sepsis incident in patients without AUR ( $p=1.000$, which was not statistically significant) (- Table $\mathbf{1}$ ).

The recatheterization rate was $23.0 \%$ in the AUR group, whereas it was only $3.8 \%$ in the non-AUR group after TURP. This difference in recatheterization rate was statistically significant $(p=0.007)$ ( - Table $\mathbf{1})$.

Eighteen patients in the AUR group developed irritative LUTS such as incontinence, increased frequency, and urgency. In the non-AUR group, only 8 patients developed irritative LUTS $(p=0.319$, which was not statistically significant) $(-$ Table $\mathbf{1})$.

In the current study, around one-fourth of the patients, that is $24.3 \%$ of patients in the AUR group, suffered from UTI after TURP. This was relatively low in patients without AUR, developed only in $3.9 \%(p=0.004$, which was statistically significant) (-Table $\mathbf{1}$ ). 
In the present study, only one patient in the AUR group required resurgery for clot retention $(p=1.000$, which was not statistically significant) (-Table $\mathbf{1}$ ).

The length of the hospital stay was statistically significant in our study, as evidenced by the $p$-value of 0.000 ( - Table $\mathbf{1}$ ).

The mean PVR in AUR and non-AUR groups were 14.31 and $13.32 \mathrm{~mL}$, respectively, it was not statistically significant. The Qmax was also almost the same between these two groups. We excluded the patients who developed recurrent retention (-Table $\mathbf{1})$.

In the current study, two patients in the AUR group and one patient in the non-AUR group developed stricture during 3 months of follow-up ( $p=1.000$, which was statistically insignificant (-Table $\mathbf{1}$ ).

\section{Discussion}

Benign prostatic hyperplasia is a common urological problem affecting men at an older age. Acute urinary retention may be the presenting symptom. The prevalence rate of AUR in men with BPH varies. In western countries, the incidence rate is lower, ranging from 20 to $40 \%$, whereas in developing countries the rate is relatively higher, reaching more than $50 \%{ }^{15}$. The increased incidence of AUR in men with BPH in developing countries is unawareness of BPH symptoms, fear of surgery, and cost factors. Chen et $\mathrm{al}^{16}$ from Taiwan conducted a retrospective study and found that post-TURP complications were more in patients who presented with AUR than in those presented without AUR.

Our study enrolled 126 patients diagnosed as BPH with their symptoms, clinical examinations, uroflowmetry, and USG. Of these, 74 patients presented with AUR, and 52 presented without AUR. We compared the following factors of preoperative variables: age, presence of any comorbid illness, gland size, the gland's grade by DRE, and serum PSA. The following postoperative variables were compared: hematuria, need for blood transfusion, UTI, sepsis, recatheterization rate, PVR, length of hospital stay, lower urinary tract stricture, resurgery rate, TUR syndrome, and Qmax.

In the current study, men aged 40 to 70 years were included. Of these, the mean age for men presenting with AUR was 62.51 years, and that in men without AUR was 61.06 years. The $p$-value for the mean age was 0.164 , which was not significant. So, both the groups were comparable in age. A study conducted by Kurita et al ${ }^{17}$ also showed no statistical difference between these two groups based on age. In comparison, other studies such as those by Kaplan et $\mathrm{al}^{18}$ and Lowe et $\mathrm{al}^{19}$ showed that AUR occurred more commonly in older age groups.

Regarding comorbid factors, HT was present in $21.6 \%$ of patients with AUR and $19.2 \%$ of patients without AUR, and the $p$-value was 0.91 , which was not significant. DM was present in $23 \%$ of patients with AUR and $23.1 \%$ of patients without AUR. The $p$-value here was 1.000 , which was not significant. Next, $10.8 \%$ of the patients with AUR and $9.6 \%$ of patients without AUR had IHD with a $p$-value of 1.000. So, in our study, both groups were comparable in comorbid illness.
A few studies have shown that the presence of co-morbid factors might be confounding factors.

Only one patient in the AUR group developed TUR syndrome immediately at the end of the procedure. It was suspected clinically, and serum electrolytes were evaluated, which showed hyponatremia, and it was corrected. No patients in the non-AUR group developed this syndrome.

In our study, $10.8 \%$ of patients with AUR and $5.8 \%$ of patients without AUR had hematuria after TURP. The $p$-value was 0.523 , which was not significant. Chen et al's ${ }^{16}$ study showed hematuria in $8.1 \%$ of patients with AUR and $7.4 \%$ of patients without AUR. Our study was more or less similar to this study. Holtgrewe et al's ${ }^{12}$ study showed hematuria and blood transfusion in $6.4 \%$ of patients, Rassweiler et al ${ }^{20}$ showed hematuria and blood transfusion in $2 \%$ of patients, whereas it was $22 \%$ in a study done by Doll et al. ${ }^{21}$

Blood transfusion rate was $4.1 \%$ and $1.9 \%$ for patients with and without AUR, respectively, with a $p$-value of 0.642 , which was not significant. Chen et al ${ }^{16}$ showed a blood transfusion rate of $3.2 \%$ and $1.5 \%$ for patients presented with and without AUR, respectively.

Only one patient (1.4\%) with AUR developed sepsis after TURP in our study. No patient without AUR had sepsis. The patient was treated intensively with IV fluids and higher antibiotics. Chen et al's ${ }^{16}$ study reported sepsis in $1.4 \%$ of patients in the AUR group. Holtgrewe et $\mathrm{al}^{12}$ and Haupt et al ${ }^{22}$ showed urosepsis in $0.2 \%$ of patients after TURP. Doll et al ${ }^{21}$ showed urosepsis in $3 \%$ of patients.

In the current study, $23 \%$ of patients with AUR developed urinary retention after catheter removal in TURP, which was quite higher when compared with $3.8 \%$ of patients without AUR. This was statistically significant with a $p$-value of 0.007 . If the patient developed urinary retention, we catheterized the patient, placed him on an $\alpha$-blocker, and provided a trial void after 1 week. All our patients responded well in trial voiding. Chen et al's ${ }^{16}$ study showed a recatheterization rate of $13.8 \%$ and $0 \%$ for patients with and without AUR, respectively. Holtgrewe et a ${ }^{12}$ showed recatheterization in $6.5 \%$ of patients, Doll et al ${ }^{21}$ in $3 \%$, and Borboroguli et a ${ }^{23}$ in $7.1 \%$ after TURP. The increased rate of recatheterization in patients with AUR may be due to hypoactive detrusor after chronic obstruction, inadequate resection due to increased gland size, or early cessation of the procedure due to patient factors.

Eighteen (24.3\%) patients in the AUR group developed irritative LUTS such as incontinence, increased frequency, and urgency. Only $8(15.4 \%)$ patients developed irritative LUTS in the non-AUR group $(p=0.319)$. The difference between the two groups was not statistically significant, as evidenced by the $p$-value of 0.319 .

We did urine culture and sensitivity for all our patients postoperatively. In our study, $24.3 \%$ of patients with AUR and only $3.9 \%$ of patients without AUR had UTI documented by urine culture. These patients were given a course of culturespecific antibiotics. UTI was higher in patients with AUR with a $p$-value of 0.004 , which was significant. This increased occurrence of UTI may be due to prolonged catheterization and hospital stay in patients with AUR. Chen et $\mathrm{al}^{16}$ reported a UTI rate of $18.6 \%$ in the AUR group and $15.6 \%$ in the non- 
AUR group. Borboroglu et $\mathrm{al}^{23}$ showed a UTI rate of $4 \%$, whereas it was relatively higher in Doll et al's ${ }^{21}$ study, which showed $25 \%$.

In our study, three patients developed lower urinary tract stricture, $2(2.7 \%)$ in the AUR group and $1(1.9 \%)$ in the nonAUR group. This was diagnosed 3 months after TURP when the patient complained of a thin stream and straining to void. We did a urethrogram for these patients and diagnosed the stricture. We advised optical internal urethrotomy for these patients. These three patients were not willing to undergo urethrotomy. Hence, dilatation was done. The reasons for the stricture formation may be due to instrumental injury, diathermy injury during TURP, or due to prolonged catheterization. Chen et al's ${ }^{16}$ study showed that patients with and without AUR showed lower urinary tract stricture rate difference of $2.6 \%$ and $3.2 \%$, respectively.

Only one patient $(1.4 \%)$ in our study developed clot retention. Cystoscopic clot evacuation was done postoperatively.

The length of stay was 7 days and 4.56 days for patients with and without AUR, respectively. This was statistically significant $(p=0.000)$.

The mean postoperative PVR for patients with and without AUR was $14.31 \mathrm{~mL}$ and $13.32 \mathrm{~mL}$, respectively. The $p$-value was 0.062 , which was not significant statistically.

We did uroflowmetry for all patients who voided after TURP to compare urine flow patterns. The mean Qmax was $19.22 \mathrm{~mL} / \mathrm{sec}$ and $19.20 \mathrm{~mL} / \mathrm{sec}$ for patients with and without AUR, respectively. The $p$-value was 0.947 , which was not significant.

\section{Conclusion}

Our study shows that post-TURP complications such as hematuria, blood transfusion rate, post-operative UTI, sepsis, recatheterization, lower urinary tract stricture, resurgery, TUR syndrome, and length of hospital stay were higher in patients who presented with AUR than in patients without AUR. Of these complications, post-TURP UTI, recatheterization rate, and the length of hospital stay were statistically significant in the AUR group compared with the non-AUR group. Therefore, it is better to intervene earlier before the patients develop AUR to minimize the complications and maximize the outcomes.

\section{Conflict of Interest}

None declared.

\section{References}

1 Reynard JM, Yang Q Donovan JL, et al. The ICS-'BPH' study: uroflowmetry, lower urinary tract symptoms and bladder outlet obstruction. Br J Urol 1998;82(05):619-623

2 Kuo HC. Pathophysiology of lower urinary tract symptoms in aged men without bladder outlet obstruction. Urol Int 2000;64(02):86-92

3 Hutchison A, Farmer R, Chapple C, et al. Characteristics of patients presenting with LUTS/BPH in six European countries. Eur Urol 2006;50(03):555-561
4 Moorthy HK, Philip S. TURP syndrome - current concepts in the pathophysiology and management. Indian J Urol 2001;17:97-102

5 McConnell JD, Bruskewitz R, Walsh P, et al. Finasteride reduces the need for surgery in men with benign prostatic hypertrophy. South Med J 1998;91(07):687

6 Tan AHH, Gilling PJ, Kennett KM, Frampton C, Westenberg AM, Fraundorfer MR. A randomized trial comparing holmium laser enucleation of the prostate with transurethral resection of the prostate for the treatment of bladder outlet obstruction secondary to benign prostatic hyperplasia in large glands (40 to 200 grams). J Urol 2003;170(4 Pt 1):1270-1274

7 Westenberg A, Gilling P, Kennett K, Frampton C, Fraundorfer M. Holmium laser resection of the prostate versus transurethral resection of the prostate: results of a randomized trial with 4-year minimum long-term followup. J Urol 2004;172(02):616-619

8 Emberton M, Anson K. Acute urinary retention in men: an age old problem. BMJ 1999;318(7188):921-925

9 Choong S, Emberton M. Acute urinary retention. BJU Int 2000;85 (02):186-201

10 Rom M, Waldert M, Klingler HC, Klatte T. Bladder outlet obstruction in men with acute urinary retention: an urodynamic study. World J Urol 2013;31(05):1045-1050

11 Pickard R, Emberton M, Neal DENational Prostatectomy Audit Steering Group. The management of men with acute urinary retention. Br J Urol 1998;81(05):712-720

12 Holtgrewe HL, Mebust WK, Dowd JB, Cockett AT, Peters PC, Proctor C. Transurethral prostatectomy: practice aspects of the dominant operation in American urology. J Urol 1989a141(02): 248-253

13 Loh SY, Chin CM. A demographic profile of patients undergoing transurethral resection of the prostate for benign prostate hyperplasia and presenting in acute urinary retention. BJU Int 2002;89 (06):531-533

14 Lodh B, Sinam RS, Singh KA. Digital rectal grading of benign prostatic hyperplasia: Where does it stand today? J Mahatma Gandhi Inst Med Sci 2016;21:40-45

15 Malone PR, Cook A, Edmonson R, Gill MW, Shearer RJ. Prostatectomy: patients' perception and long-term follow-up. Br J Urol 1988;61(03):234-238

16 Chen JS, Chang CH, Yang WH, Kao YH. Acute urinary retention increases the risk of complications after transurethral resection of the prostate: a population-based study. BJU Int 2012;110(11 Pt C):E896-E901

17 Kurita Y, Masuda H, Terada H, Suzuki K, Fujita K. Transition zone index as a risk factor for acute urinary retention in benign prostatic hyperplasia. Urology 1998;51(04):595-600

18 Kaplan SA, Wein AJ, Staskin DR, Roehrborn CG, Steers WD. Urinary retention and post-void residual urine in men: separating truth from tradition. J Urol 2008;180(01):47-54

19 Lowe FC, Batista J, Berges R, et al. Risk factors for disease progression in patients with lower urinary tract symptoms/ benign prostatic hyperplasia (LUTS/BPH): a systematic analysis of expert opinion. Prostate Cancer Prostatic Dis 2005;8(03): 206-209

20 Rassweiler J, Teber D, Kuntz R, Hofmann R. Complications of transurethral resection of the prostate (TURP)-incidence, management, and prevention. Eur Urol 2006;50(05):969-979

21 Doll HA, Black NA, McPherson K, Williams GB, Smith JC. Differences in outcome of transurethral resection of the prostate for benign prostatic hypertrophy between three diagnostic categories. Br J Urol 1993;72(03):322-330

22 Haupt G, Pannek J, Benkert S, Heinrich C, Schulze H, Senge T. Transurethral resection of the prostate with microprocessor controlled electrosurgical unit. J Urol 1997;158(02):497-501

23 Borboroglu PG, Kane CJ, Ward JF, Roberts JL, Sands JP. Immediate and postoperative complications of transurethral prostatectomy in the 1990s. J Urol 1999;162(04):1307-1310 